\title{
A Progressive Communication with Multi-Source Fusion for the Disabled
}

\author{
Shin-Sook Yoon ${ }^{1}$, Dong-Hyeok $\mathrm{Suh}^{2}$, Chang-Won $\mathrm{Seo}^{3}$, Sei-Cheol $\mathrm{Oh}^{4}$ \\ and Keun-ho Ryu ${ }^{1}$ \\ ${ }^{1}$ Chungdae-ro, Seowon-gu, Cheongju-si, Chungbuk, 28644, Korea \\ ${ }^{2} 119$, Dandae-ro, Dongnam-gu, Cheonan-si, Chungnam, 31116, Korea \\ ${ }^{3} 76-32$, Daehakgil, Kamgok-myun, Eumsung-gun, Chungbuk, 27601, Korea \\ ${ }^{4} 48$, Weolbong-ro, Seobuk-gu, Cheonan-si, Chungnam, 31172, Korea \\ 1yss28@daum.net, ${ }^{2}$ dhsuh122@dankook.ac.kr, ${ }^{3}$ cwseo@kdu.ac.kr, \\ ${ }^{4}$ scoh@kornu.ac.kr, ${ }^{1}$ khryu@chungbuk.ac.kr
}

\begin{abstract}
A variety of communication methods for people with communicative disorders, who have difficulties in communication have been studied. For people who have difficulties in recognizing voice, methods to recognize meaning that a speaker attempts to provide, by recognizing motion of his/her lip and those to use perceived gestures or talking with the hands in communication have been studied. This study proposed a plan to help communication of people with communicative disorders, by using the state estimation which is often used when IoT converges data with multi-sensors. It also provided a plan to quickly enable communication through other alternatives, when stability and reliability of main communication are decreased, by inspecting states of the existing communication. More stable communicative alternatives are substituted, if the stability of communication is lost due to environmental or conditional effects, after designing main communicative methods depending on types of disables. Specific plans to evaluate the stability of communication are suggested by this study. Although the theory of evidence was designed for mathematically expressing ambiguity of real world, methods to estimate the current state of system, caused by many factors and a factor that has most significant effects on the state are provided. This study adopted and used the state estimation originated from the theory of evidence, in order to estimate the stability.
\end{abstract}

Keywords: disability, Communication, Internet of Thing, Sensor Data, Emergency situation.

\section{Introduction}

The communication is very important for human life. When it is disrupted, an unnecessary waste of resources is inevitable, and assets possessed by individuals and groups cannot be efficiently spent. Fluent communication promotes national and social productive activities and increase of assets. It enables various exchanges and enriches trade. The limit of distance in communication between human beings is markedly lowered, through the development of communication. The continuous development of it enlarges life areas and allows usage of possessed goods to be expanded, by increasing exchanges among human beings and groups.

The communication basically requires terminals, channels and protocols. In the real world, communication channels are faced with many limits. It costs to organize the channels and noises can be added to them even thought they are established.

Received (November 21, 2017), Review Result (January 15, 2018), Accepted (January 31, 2018) 
Moreover, even contents of communication may be often distorted, because of problems of the channels.

The most common channel between human beings is voice communication, through air. The problem of voice communication is that it is seriously disrupted even though a distance between two people is a little increased. The voice communication also meets with serious obstacles, if noises around it are increased. Human beings have invented various kinds of communication systems, to overcome such problems and enable fluent communication. A variety of methods for voice communication between human beings aim to overcome the distance limit and control addition of noises and occurrence of distortion.

On the other hand, newly developed methods are applied to communication in human society. The communication has been traditionally conducted among users who possess communication terminals. It tends to be recently conducted, as information process devices occupy terminals or connect to them. In such a communication system, contents distributed through communication channels are often digital data with accumulated with various signals and figures, rather than voice information.

Data communication is also dramatically advanced in these days, and $\mathrm{M} 2 \mathrm{M}$ is quietly highlighted. M2M means machine-to-machine or machine-to-man. It essentially implies that it is possible to maintain and manage communication channels and the entire communication, where no human beings are required for the communication system. Thus, M2M serves a core role in communication network of IoT.

IoT transmits context-information of the real world to users via servers, by using multi-sensors. Some clues for various alternative communication can be acquired from methods to transmit context-information gained by different multi-sensors to users.

Meanwhile, such communication methods can be useful for people who experience difficulties in their communication.

Sometimes people with disabilities have difficulty communicating. For this reason, communication between disabled and non-disabled people is difficult. In the case of using speech even in the communication of the non-disabled, communication difficulties may occur depending on the surrounding environment.

A variety of studies on communication of people with disabilities have been conducted. Among them, the representative one examines lip recognition.

Communication through lip recognition is beneficial as a communication tool for people who are difficult to recognize speech. Communication through lip recognition is not only for the handicapped, but also complementing the problems of speech recognition. Communication through voice transmission has a problem in that recognition becomes difficult when the surrounding noise is large.

By adopting the visual information transmission method, it is possible to enhance the stability of information transmission in an environment where voice recognition is difficult.

Furthermore, using a combination of different methods or using a combination of the most effective ones rather than using a single method for communication can clarify information transmission.

In this case, the voice signal, the image data, and the motion recognition sensor values have different properties, and therefore, a method capable of fusion is needed.

This study proposes an evaluation method for each factor for multi - expression convergence processing that helps communication between disabled and non disabled people. 
Synchronization schemes are required for different data, but this study assumes that synchronization is smooth. Eliminating uncertainty in communication will help people with communication difficulties. There are many cases in which nondisabled persons may have difficulty in communication depending on the surrounding environment and conditions.

Therefore, what we are trying to solve in this study could provide benefits for both the disabled and the non-disabled.

This paper is composed as follows. Section 2 summarizes multi - information convergence and Section 3 proposes a detailed element evaluation method to support multi - expression convergence for improved communication.

In Section 4, experiments and evaluations are carried out and conclusions are made in Section 5 .

\section{Related Research}

There have been many studies to recognize target-situations based on measurements provided by sensors, since ubiquitous networking received attention, before IoT appears. Some figures detected and reported by sensors were transmitted, stored and processed in the form of data. Here, data collected by sensors undergo some preprocessing steps: data filtering through which only changes detected as significant ones are reported; data aggregation which keeps assets of network system from being wasted by eliminating sensored duplication of data; and data merge or data fusion which enables fusion of any data reported by heterogeneous sensors. The multi-sensor data fusion processing for acquring better contextinformation has attracted much attention from researchers, among processing of such sensor data. The use of multi-sensors can make the accuracy of information stronger than estimation by using data detected by a single sensor, so many researchers have concentrated on such studies. Although ubiquitous sensor networks has not been put to practical use, due to their faults, they played a good role in providing an opportunity to seek out methods to use measurements acquired by sensors constituting sensor networks. By the age of IoT, the instability of ubiquitous sensor network system is substantially improved.

Multi-signal or multi-data fusion begins with the need to overcome the limitations of a single sensor. There are various kinds of sensors that detect phenomena in the real world. Each sensor can detect physical, chemical, or biological changes and convert these changes into numbers.

When sensors are of different kinds, the values sensed and marked by each sensor are completely different from each other. The fusing process is aimed at values measured by sensors that are different from each other.

Multi-expression convergence processing is based on the technology called Data Fusion, Data Merge in the wireless sensor network age. Data fusion has already begun in the 1960s. The initial data fusion process was used to estimate the trajectory of a moving object in a linear system. The signal data from different radars were fused to calculate the trajectory of the launch vehicle[1][2].

The method used here is Kalman Filter. Since then Kalman Filter has developed to a level that can be applied to non-linear moving objects[3].This method can be used to estimate the position or state of a target after a certain period of time by fusion processing heterogeneous sensor values[4].

The Bayesian theorem and the Dempster-Shafer proof theory are probabilistic theories that can be used to fuse heterogeneous values[5]. While the Bayesian theorem has the constraint of knowing the probability of each case in advance, Dempster-Shafer proof theory can not only represent the real-world ambiguity but also integrate heterogeneous elements. In the proof theory, the basic probability 
assignment function is the core, and it is important to appropriately assign the probability assignment function to the number of various cases in which the elements to be fused are combined[6][7].

By computing the basic probability assignment function assigned to the focal elements belonging to the power set, it is possible to mathematically represent the events of the real world and can also be used for convergence processing. In addition, there is a method of fusion of heterogeneous elements using artificial neural network. The characteristics of the method using the artificial neural network go through a machine learning process. This feature may cause some inconvenience to the real-time processing that reacts quickly.

There is a way to fuse using fuzzy theory. It is necessary to define fuzzy function. However, when fuzzy theory is used, it has good scalability and less computational complexity than other methods.

\section{A Way of Multi-Source Fusion for Progressive Communication of the Disabled}

The communication can be disrupted due to many reasons. Communication through elaborate and durable systems often has high reliability. However, communication between human beings tend to have low reliability.

This study has intention to support a bottleneck of communication among people with life difficulties, by using a procedure used for multi-sensor data fusion processing. Many communication methods between human beings are conducted by using voice, talking with the hands and lip recognition. This paper suggests that a main communication method is initially used, and then, replaced with an alternative one, if an error of communication occurs, in order to continue fluent communication.

Data fusion combines heterogeneous data. Heterogeneity is based on physical heterogeneity. What you can expect when merging heterogeneous signals or data is the quality improvement of information. Data convergence can be used for communication, when communication channels are diverse. That is, data convergence can be attempted in an environment capable of communicating with various channels such as voice signals, image information, and sensor data. It may be difficult for people with disabilities to use communication channels that non-disabled people can use on a daily basis.

This study presupposes an environment in which communication channels can be communicated in various ways with the disabled with difficulties in communication.

In order to communicate the handicapped, this study proposes the following communication algorithm.

1) Use voice transmission, image information, and sensor data for communication.

2) The main communication method is voice transmission.

3) Check the clarity of the communication signal by setting a constant time interval according to the time.

4) Main communication channel is decided according to user's condition first. For example, a normal person first checks the state of voice transmission. The hearing impaired person sets a communication channel other than the voice transmission state as the main channel.

5) Check the main communication channel first.

6) The voice transmission state checks the voice state of the information source and the ambient noise state, respectively.

7) Dempster-Shafer Evidence Theory (DST) is used to assess the state of voice transmission.

8) Check the reliability and uncertainty of voice signal transmission. 
9) When uncertainty grows and reliability declines, switch to another communication channel.

10) Check the status of other communication channels, and select channels with high reliability and low uncertainty.

The following is a suggestion to check the status of each communication channel. In order to check the status of each communicating channel, we evaluated the voice transmission channel in this study. As an evaluation factor, the recognition of the sound size, the noise of the space, and the appearance frequency of the moving object in the space sensed by the acoustic sensor were made.

These three factors are factors to be included in the state calculation of the communication channel. These three factors are evaluated and the results are averaged to provide inferences about the state of speech transmission in the target space. At this time, the above three factors are regarded as three pieces of evidence related to the communication situation, and these three factors can be merged and used for checking the communication state in the space.

The Dempster-Shafer Evidence Theory(DST) is useful for communicative reasoning by this calculation. The key to convergence processing using DST is to compute a basic probability assignment function. The DST-based data convergence method for inference of the communication situation and the method of calculating the basic probability assignment function necessary for the situation inference are expressed as follows.

$$
p_{i \cdot t}=\frac{x_{i \cdot t}}{\sum_{t=1}^{n} x_{i \cdot t}}
$$

$x_{t}=$ impiedelements, $S_{k \cdot t}=\sum n$, (window),$n=$ window,$i=$ element number (its number is $2^{m}-1$ because of the number of subset), $m=$ number of sensors.

$$
\dot{p}_{i}=\frac{\sum_{t=1}^{n} p_{i \cdot t}}{n}
$$

Through the basic probability assignment function, it is possible to infer the situation by fusing the measured values detected by each sensor.

Table 1. Psudocode

\#start of the algorithm

Start and Initiallization

Input: voice_data, image_data, sensor_data,current_time, user_input, threshold

Output: A channel with high reliability and low uncertainty

\section{\#define functions}

get_data(source):

$\mathrm{t} \_0=($ current_time $)$

while $\mathrm{t}<$ size(timeslot):

$\mathrm{t}=$ (current_time) $-\mathrm{t} \_0$

Dataset $=$ Dataset.append $($ Data_of_source $($ current_time $))$

return dataset 
check_state(dataset):

if Noise(dataset)) > threshold:

return "source is in the noise state"

else

return "source is in the clear state"

DST(dataset):

$\mathrm{t} 1=0$

while $\mathrm{t} 1<\mathrm{n}$ :

$\mathrm{t} 2=0$

while $\mathrm{t} 2<\mathrm{n}$ :

Sum $=$ Sum $+x_{i \cdot t 2}$ $\mathrm{t} 2=\mathrm{t} 2+1$

$p_{i \cdot t 1}=x_{i \cdot t 1} /$ Sum

Psum $=$ Psum $+p_{i \cdot t 1}$

$\dot{p}_{i}=$ Psum $/ \mathrm{n}$

rel $=$ calculate_reliability $\left(\right.$ dataset, $\left.p_{i}\right)$

unc $=$ calculate_uncertainty $\left(\right.$ dataset,$\left.p_{i}\right)$

return (rel, unc)

\#body of the algorithm

While True:

ifuser_input $==$ stop_command:

break

else

Channel $=$ Default $\_$channel $($voice $)$

DS_v = get_data(voice_data)

DS_i $=$ get_data(image_data)

DS_s $=$ get_data(sensor_data)

for dataset in (DS_v, DS_i, DS_s)

$\operatorname{val}[$ dataset $]=\mathrm{DST}$ (dataset)

print(check_state(dataset))

if $\frac{\mathrm{d}}{\mathrm{dt}}$ unc $($ Channel $)>0 \& \& \frac{\mathrm{d}}{\mathrm{dt}} \operatorname{rel}($ Channel $)<0$ :

for dataset inval[DS_v], val[DS_i], val[DS_s] :

chan $1=$ find channel of $\min (\mathrm{unc})$ or $\max (\mathrm{rel})$

switch Channel to chan1

End \#end of the algorithm 
The state estimation is important for methods suggested by this chapter. The key idea is that a main communication channel is selected and used for communication, but when it is disrupted, an alternative channel should be operated to substitute the main channel, in order to keep fluent communication. Here, it is important to estimate stability and states of the main communication channel. Alternative methods to keep the reliability of communication can be conducted, only if communication states which contribute to stability of communication line are rightly understood. Methods suggested in this chapter can be expressed as virtual codes.

This chapter argues that reasonable state estimation be conducted, in frequently checking states of main communication channels which is previously selected, and suggests that communication methods be turned to more definite ones, after verifying outcomes of state estimation.

In Chapter 4, we test the proposed method in this chapter

\section{Experiment and Evaluation}

In this chapter, the experiment was carried out as follows. An acoustic sensor was installed in the target space to detect the transmission state of the voice signal, to detect the ambient noise state, and to detect the entrance / exit situation of the moving object in the space by arranging the ultrasonic sensor.

We calculate the pseudo state according to the proposed method in Section 3 with the size of the sound and the situation data recognized by each sensor. The results are summarized in the following table.

In this way, the communication state is calculated by integrating the reliability of each of the three factors. If the sensors report partial evidence of the situation, they can be inferred from the history of the situation by converging them.

Table 1 shows the results of inferring the situation using the basic probability assignment function. Table 1 shows the reliability and probability values of each element using the basic probability assignment function.

The reliability of the situation is calculated through the above experimental results, and it is possible to deduce what aspect each situation has and why it is dangerous situation. In Table 1, bel values indicate reliability. The $\mathrm{pl}$ value is indicative of plausibility. It is uncertainty that reliability is deducted from the probability. $\mathrm{h} 1$ is the voice transmission state, $\mathrm{h} 2$ is the ambient noise state, and $\mathrm{h} 3$ is the moving state of the moving object.

Table 1. Communication State Inference in Target Space

\begin{tabular}{llll}
\hline & $\mathrm{h} 1$ & $\mathrm{~h} 2$ & $\mathrm{~h} 3$ \\
\hline bel & 0 & 0.4798 & 0.1049 \\
\hline $\mathrm{pl}$ & 0.2289 & 0.8687 & 0.4001 \\
\hline $\mathrm{pl}-\mathrm{bel}$ & 0.2289 & 0.3889 & 0.2951 \\
\hline bel & 0.0004 & 0.5967 & 0.0489 \\
\hline $\mathrm{pl}$ & 0.2208 & 0.9377 & 0.2544 \\
\hline $\mathrm{pl}-\mathrm{bel}$ & 0.2204 & 0.3410 & 0.2054 \\
\hline bel & 0 & 0.2120 & 0.4313 \\
\hline $\mathrm{pl}$ & 0.2210 & 0.4608 & 0.7349 \\
\hline $\mathrm{pl}-\mathrm{bel}$ & 0.2210 & 0.2488 & 0.3036 \\
\hline
\end{tabular}

The result of the experiment and calculation shown in the above table show the reliability of the voice transmission state is 0 in the first situation. The ambient noise has reliability close to 0.5 . The entry / exit status of the moving object is 0.1 . 
This result implies that voice transmission is not suitable for use as a communication channel.

In the second situation, the reliability of the noise condition is still high. However, in the third situation, the reliability of the noise is remarkably reduced and the movement of the moving object is increased.

This experiment confirms that the inadequate factors for using voice transmission as a channel are the increase of ambient noise and moving objects.

\section{Conclusion}

The communication has made a significant contribution to prosperity and development of the human society. The human society has continued very notable development. The communication consists of terminals, channels and protocols. It has been advanced by minimizing noises introduced through terminals or channels and resolving signal distortion, and further developed to reduce all kinds of loss in the process of transmission. When communication is not fluent or failed, users cannot but sustain extreme inconvenience and loss.

This study attempted to ensure the reliability of communication by used the state estimation, among technologies for multi-sensor fusion procession used in IoT and enabling adaptive selection of communication channels and methods.

IoT has implemented intellectual communication methods, different from the existing data communication. IoT can provide high-quality context-information by transmitting data detected by various sensors to users. It shows that it is possible to support communication of people with disabilities, who have difficulties in communication, by putting IoT's context-information transmission schemes to practical use.

People with disabilities are experiencing various types of life difficulties. Such disabilities include inborn and acquired disabilities due to accidents or diseases. People with disabilities are categorized depending on types of disabilities, however, there is a little difference in criteria for categorization, between education and welfare support. People with physical disabilities have difficulties in movements or behaviors in life spaces, while people with communicative disorders are not able to speak and hear, or have substantial difficulties in speaking and hearing. In order to enable communication of people with communicative disorders, some measures including use of a hearing aid and talking with the hands have been developed. It can be possible to seek out various supports for communication, as information and communication technology has been recently developed.

In this study, we can support the communication of disabled people through various communication methods using various channels. In this case, we provide the basis of judgment to switch to other communication channels by checking the reliability of each channel at certain time intervals.

The Dempster-Shafer theory of evidence was originally designed to indicate ambiguity of the real world through mathematical probability. A lot of researchers have used the theory of evidence for fusion of heterogeneous data. This study attempted to use it for the state estimation. To this end, it was used as evaluative calculation for each of factors and a combination of factors.

DST was used to converge heterogeneous multiple data, and the confidence interval and uncertainty interval for each factor were calculated and evaluated.

It can be applied to multi - channel communication by using the result of calculation. When main communication channel exposes a problem, other communication channel can be actively selected to supplement it. 


\section{Acknowledgments}

This work was supported by the Ministry of Education of the Republic of Korea and the National Research Foundation of Korea(NRF-2016S1A3A2925520)

This paper is a revised and extended version of a paper entitled [A Novel Way of Multi-Expression Fusion for Enhanced Communication of the Disabled] presented at [FGIT2017, Daejeon University, Korea, December21-23, 2017].

\section{References}

[1] L. A. Klein, "Sensor and data fusion: A tool for information assessment and decision making", SPIE Press, (2004).

[2] B. Khaleghi, A. Khamis, F. O. Karray and S. N. Razavi, "Multi sensor data fusion: A review of the state-of-the-art", Information Fusion, vol.14, issue 1, (2013), pp.28-44.

[3] S.L. Sun and Z.L. Deng, "Multi-sensor optimal information fusion Kalman filter", Automatica, vol. 40,(2004), pp.1017-1023.

[4] Y. R. Kim, S. K. Sul and M. H. Park, "Speed Sensorless Vector Control of Induction Motor Using Extended Kalman Filter", IEEE Transactions on industry applications, vol. 30, no. 5, (1994), pp.12251233.

[5] D.Y. Ahn, S. G. Shin, J. O. Lee and H. K. Lee, "Object Identification Strategy in the AEB Pedestrian System Using the Dempster-Shafer Theory", The Korea of Society Automotive Engineers, vol. 2016, no.5, (2016), pp. 1285-1290.

[6] D. Y. Ahn, S. G. Shin and H. K. Lee, "Improvement of Pedestrian Recognition Rate of Sensor Fusion Using the Dempster-Shafer Theory", The Korea of Society Automotive Engineers. vol.2015, no.5, (2015), pp. 623-627.

[7] A. P. Dempster, "Upper and Lower Probabilities Induced by a Multivalued Mapping", Annals of Mathematical Statistics, 38, (1967), pp. 325-339.

\section{Authors}

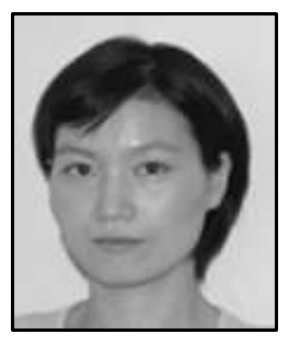

Shin-Sook Yoon, she received the M.S. degrees in computer engineering from Hoseo University in 2008 and the Ph.D. Candidate in computer science from Chungbuk National University in 2011. Her research interests included in Bioinformatics and data fusion in wireless sensor network.

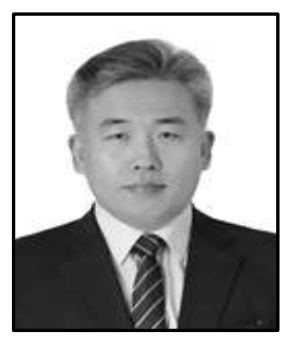

Dong-Hyeok Suh, he is a professor at Dankook University. He received the M.S. degrees in computer engineering from Hoseo University in 2005 and the Ph.D. in computer science from Chungbuk National University in 2012. His research interests included in stream data processing and data fusion in wireless sensor network.

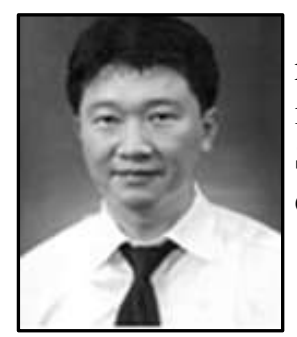

Chang-Won Seo, he is a professor at Far East University. He received the B.A. degree in special education from Taegu university in1994 and the M.A. degree in 2002. He received the Ph.D. from Seoul National University. His research interests included communication tool. 


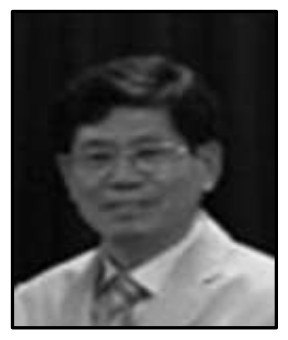

Sei-Cheol Oh, he is a professor at Korea Nazarene University. He received the B.A. degree in Arts erar from Daegu University in 1983, and the M.A. and Ph.D. in Arts from Daegu University in 1986, 1995. His research interests are included in special education and physical \& multipledisabilities.

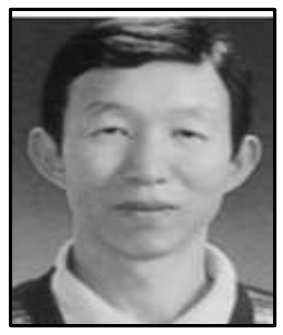

Keun-ho Ryu, he is a professor at Chungbuk National University. $\mathrm{He}$ received the M.S. degree in Computer science from Yonsei University in 1980 and the Ph.D. in 1988. He conducted research staff in computer science from University of Arizona in 1989 1991. His research interests included Spatio-temporal database application, data mining application about GIS, Digital Map, LBS, telematics, Bioinformatics analysis, medical informatics application etc. 